\title{
Analysis of Impatient Customers in Queues with Bernoulli Schedule Working Vacations and Vacation Interruption
}

\author{
Veena Goswami \\ School of Computer Application, KIIT University, Bhubaneswar 751024, India \\ Correspondence should be addressed to Veena Goswami; veena_goswami@yahoo.com
}

Received 9 July 2014; Accepted 7 September 2014; Published 21 September 2014

Academic Editor: Yurong Liu

Copyright (C) 2014 Veena Goswami. This is an open access article distributed under the Creative Commons Attribution License, which permits unrestricted use, distribution, and reproduction in any medium, provided the original work is properly cited.

\begin{abstract}
This paper analyzes customers' impatience in Markovian queueing system with multiple working vacations and Bernoulli schedule vacation interruption, where customers' impatience is due to the servers' vacation. During the working vacation period, if there are customers in the queue, the vacation can be interrupted at a service completion instant and the server begins a regular busy period with probability $1-q$ or continues the vacation with probability $q$. We obtain the probability generating functions of the stationary state probabilities and deduce the explicit expressions of the system sizes when the server is in a normal service period and in a Bernoulli schedule vacation interruption, respectively. Various performance measures such as the mean system size, the proportion of customers served, the rate of abandonment due to impatience, and the mean sojourn time of a customer served are derived. We obtain the stochastic decomposition structures of the queue length and waiting time. Finally, some numerical results to show the impact of model parameters on performance measures of the system are presented.
\end{abstract}

\section{Introduction}

Queueing systems with customers' impatience and server vacations have been analyzed due to their wide applications in real-life congestion problems such as computer and communication systems, telecommunication systems, traffic systems, service systems, and manufacturing and production systems. Impatience is the most noted feature as individuals always feel worried and impatient throughout when waiting for service which leads to loss of potential customers. It has special implication for the business world as it has an adverse impact on the revenue generation of a business organization. The customer's impatient behavior should be needed in the study of queueing system to model real conditions precisely. Occasional operation of a service may be economically invoking when entire time service would result in substantial server idle time or would prevent the utilization of the server in different productive capacities. On the other hand, the server remaining not working for periods of time might gain the probability of customer losses due to balking and reneging.

Vacation queues have been examined extensively in Ke et al. [1], Tian and Zhang [2], and so forth. Yue et al. [3] studied an $M / M / 1 / N$ queueing system with balking, reneging, and server vacations. There are several situations where the server stays active during the vacation period. The server can provide service at a lower speed during the working vacation (WV) period instead of stopping service completely. If the queue is empty at the end of a vacation, the server takes another vacation; otherwise a service period commences with normal service rate. This policy is introduced by Servi and Finn [4] and known as multiple working vacations (MWV). The stochastic decomposition forms of the waiting time and queue length in an $M / M / 1 / \mathrm{WV}$ queue have been established in Liu et al. [5]. Intending to use the server efficiently, vacation interruption has become a significant aspect. Queueing systems with vacation interruption and its variations have been investigated by Baba [6], Chen et al. [7], Li and Tian [8], and Zhang and Hou [9]. Zhang and Shi [10] presented an $M / M / 1$ queue with Bernoulli schedule vacation and vacation interruption.

There are scenarios where the customer impatience is due to the server vacation and independent of the customers in system. Queueing models with system disasters and impatient customers when system is down have been studied in Yechiali [11]. Altman and Yechiali [12] considered 
the impatience of customers only when the servers are on vacation and unavailable for service. Perel and Yechiali [13] presented queues in a fast and slow Markovian random environment, with impatient customers. Extensive analysis of queueing systems with reneging has been studied in Bocquet [14]. Laxmi et al. [15] presented $M / M / 1 / N$ working vacations queue with balking and reneging. Yue et al. [16] studied customers' impatience in an $M / M / 1$ queue with working vacations. Selvaraju and Goswami [17] analyzed impatient customers in a single server Markovian queue with single and multiple working vacations.

In this paper, we analyze impatient customers in an $M / M / 1$ queueing system with multiple working vacations and Bernoulli-schedule vacation interruption. As the server works at a slow service rate during the working vacation, customers become impatient due to this slow service rate. Under the working vacation interruption policy, at the service completion epoch the vacation is interrupted and the server switches to a regular busy period if there are customers in the queue with probability $1-q$ or remains in the vacation with probability $q$. We derive an analytic expression for the stationary distribution of probability generating functions of the number of customers in the system when the server is in a normal service period and in a Bernoulli schedule vacation interruption, respectively. Some performance measures such as mean system size, the proportion of customers served, the rate of abandonment due to impatience, and the mean sojourn time of a customer served have been evaluated. We demonstrate the stochastic decomposition structures of the stationary queue length and waiting time. Numerical results have been illustrated in the form of tables and graphs.

The rest of the paper is organized as follows. Section 2 presents the model description. In Section 3, we obtain the stationary analysis of the system and deduce the probability generating functions of the number of customers in the system when the server is in a normal service period and in a Bernoulli schedule vacation interruption, respectively. We carry out the explicit expressions for various performance measures, including the mean system size, the proportion of customers served, the rate of abandonment due to impatience, and the mean sojourn time of a customer served. The stochastic decomposition structures of the stationary queue length and waiting time are presented in Section 4. Some numerical results are given in Section 5. Section 6 concludes the paper.

\section{Model Description}

We consider the multiple working vacations $M / M / 1$ queueing system with Bernoulli schedule vacation interruptions where the customers become impatient due to the servers' vacation. Customers arrive according to a Poisson process with arrival rate $\lambda$. The service times during a normal service period, the service times during a working vacation period, and the vacation times are exponentially distributed with rates $\mu, \eta$, and $\phi$, respectively.

The customers are assumed to be impatient during the multiple working vacations. Whenever a customer arrives at the system and notices that the system is on working vacation, the customer activates an impatient timer $T$, which is exponentially distributed with rate $\xi$. If the server finishes the working vacation before the impatience timer expires, the customer remains in the system till his service completion. However, if the impatience timer expires when the server is still on working vacation, the customer abandons the system and never returns. During the working vacation period, a customer is serviced at a lower rate, and, at the instants of the service completion, the vacation is interrupted and the server resumes a regular busy period with probability $1-q$ (if there are customers in the queue) or remains in the vacation with probability $q$. The interarrival times, service times, vacation duration times, and the impatient times all are taken to be mutually independent. The customers are served on a firstcome first-served (FCFS) queue discipline.

Let $N(t)$ denote the number of customers in the system at time $t$, and let $J(t)$ denote the state of the server at time $t$ with

$$
J(t)= \begin{cases}0, & \text { if the server is in working vacation period, } \\ 1, & \text { if the server is in normal busy period. }\end{cases}
$$

Then, the pair $\{(N(t), J(t)) ; t \geq 0\}$ defines a continuous-time Markov process with state space $E=[\{(0,0) \cup(i, j)\}, i=$ $1,2, \ldots, j=0,1]$. Let $\pi_{i, j}=\lim _{t \rightarrow \infty} P\{N(t)=i, J(t)=j\}$, $(i, j) \in E$. Let $\pi_{i, 0}, i \geq 0$, be the probability that there are $i$ customers in the system when the server is in working vacation period and let $\pi_{i, 1}, i \geq 1$, be the probability that there are $i$ customers in the system when the server is in normal busy period.

\section{Stationary Probabilities}

In this section, we study a stationary analysis for the model described in the last section. We first develop the probability generating functions of the number of customers in the system when the server is in a working vacation period and in a normal service period, respectively. Then, we derive the explicit expressions for various performance measures. Using the Markov process theory, we obtain the following set of steady-state equations:

$$
\begin{gathered}
\lambda \pi_{0,0}=(\xi+\eta) \pi_{1,0}+\mu \pi_{1,1}, \\
(\lambda+\eta+\phi+n \xi) \pi_{n, 0}=\lambda \pi_{n-1,0}+(q \eta+(n+1) \xi) \pi_{n+1,0}, \\
n \geq 1,
\end{gathered}
$$

$$
\begin{gathered}
(\lambda+\mu) \pi_{1,1}=\phi \pi_{1,0}+\bar{q} \eta \pi_{2,0}+\mu \pi_{2,1}, \\
(\lambda+\mu) \pi_{n, 1}=\phi \pi_{n, 0}+\lambda \pi_{n-1,1}+\mu \pi_{n+1,1}+\bar{q} \eta \pi_{n+1,0}, \\
n \geq 2,
\end{gathered}
$$

where $\bar{q}=1-q$. Let us define the probability generating functions (pgfs) as

$$
\Pi_{0}(z)=\sum_{n=0}^{\infty} \pi_{n, 0} z^{n}, \quad \Pi_{1}(z)=\sum_{n=1}^{\infty} \pi_{n, 1} z^{n},
$$


where $\Pi_{0}(1)+\Pi_{1}(1)=1$ and $\Pi_{0}^{\prime}(z)=\sum_{n=1}^{\infty} n z^{n-1} \pi_{n, 0}$. Multiplying the appropriate power of $z^{n}$ in (2) and (3) and summing over all possible values of $n$ yield

$$
\xi z(1-z) \Pi_{0}^{\prime}(z)+g(z) \Pi_{0}(z)+A_{1} z-A_{2}(1-z)=0
$$

where

$$
\begin{gathered}
g(z)=\lambda z^{2}-(\lambda+\eta+\phi) z+q \eta \\
A_{1}=\mu \pi_{1,1}+(\phi+\bar{q} \eta) \pi_{0,0}+\bar{q} \eta \pi_{1,0}, \quad A_{2}=q \eta \pi_{0,0} .
\end{gathered}
$$

Similarly, multiplying (4) and (5) with appropriate power of $z^{n}$ and summing over $n$, we get

$$
\begin{aligned}
& (\lambda z-\mu)(1-z) \Pi_{1}(z) \\
& \quad=(\phi z+\bar{q} \eta) \Pi_{0}(z)-A_{1} z-\bar{q} \eta(1-z) \pi_{0,0} .
\end{aligned}
$$

Now, we solve the differential equation (7) in the following subsection.

3.1. Solution of the Differential Equation. Equation (7) can be written as

$$
\begin{aligned}
\frac{d}{d z} & \Pi_{0}(z)+\left\{-\frac{\lambda}{\xi}+\frac{q \eta}{\xi} \frac{1}{z}-\frac{(\phi+\bar{q} \eta)}{\xi} \frac{1}{1-z}\right\} \Pi_{0}(z) \\
& =-\frac{1}{\xi}\left(\frac{A_{1}}{1-z}-\frac{A_{2}}{z}\right),
\end{aligned}
$$

for $\xi \neq 0, z \neq 0$, and $z \neq 1$. To solve the first order linear differential equation (11), we obtain an integrating factor (IF) as

$$
\begin{gathered}
e^{\int[-\lambda / \xi+(q \eta / \xi)(1 / z)-((\phi+\bar{q} \eta) / \xi)(1 /(1-z))] d z} \\
=e^{-(\lambda / \xi) z} z^{q \eta / \xi}(1-z)^{(\phi+\bar{q} \eta) / \xi} .
\end{gathered}
$$

Multiplying both sides of (11) by IF, we get

$$
\begin{aligned}
\frac{d}{d z} & {\left[e^{-(\lambda / \xi) z} z^{q \eta / \xi}(1-z)^{(\phi+\bar{q} \eta) / \xi} \Pi_{0}(z)\right] } \\
& =-\frac{1}{\xi}\left(\frac{A_{1}}{1-z}-\frac{A_{2}}{z}\right) e^{-(\lambda / \xi) z} z^{q \eta / \xi}(1-z)^{(\phi+\bar{q} \eta) / \xi} .
\end{aligned}
$$

Integrating both sides of (13) from 0 to $z$, we get

$$
\Pi_{0}(z)=\frac{-A_{1} K_{1}(z)+A_{2} K_{2}(z)}{\xi e^{-(\lambda / \xi) z} z^{q \eta / \xi}(1-z)^{(\phi+\bar{q} \eta) / \xi}},
$$

where

$$
\begin{aligned}
& K_{1}(z)=\int_{0}^{z} e^{-(\lambda / \xi) x} x^{q \eta / \xi}(1-x)^{(\phi+\bar{q} \eta) / \xi-1} d x, \\
& K_{2}(z)=\int_{0}^{z} e^{-(\lambda / \xi) x} x^{q \eta / \xi-1}(1-x)^{(\phi+\bar{q} \eta) / \xi} d x .
\end{aligned}
$$

Remark 1. Setting $\eta=0$ in (7) yields

$$
\begin{aligned}
\xi(1-z) \Pi_{0}^{\prime}(z)= & (\lambda(1-z)+\phi) \Pi_{0}(z) \\
& -\left(\mu \pi_{1,1}+\phi \pi_{0,0}\right),
\end{aligned}
$$

which matches with Altman and Yechiali [12].

Remark 2. Setting $q=1$ in (7), we get

$$
\begin{aligned}
& \xi z(1-z) \Pi_{0}^{\prime}(z)+\left(\lambda z^{2}-(\lambda+\eta+\phi) z+\eta\right) \Pi_{0}(z) \\
& \quad+z\left(\mu \pi_{1,1}+\phi \pi_{0,0}\right)-\eta(1-z) \pi_{0,0}=0,
\end{aligned}
$$

which agrees with Yue et al. [16].

Remark 3. Setting $\xi=0$ and $q=1$ in (7), we get

$$
\Pi_{0}(z)=\frac{\eta(1-z) \pi_{0,0}-z\left(\mu \pi_{1,1}+\phi \pi_{0,0}\right)}{\lambda z^{2}-(\lambda+\eta+\phi) z+\eta} .
$$

The above result matches with Servi and Finn [4].

It is seen from (14) that $\Pi_{0}(z)$ is expressed in terms of $\pi_{0,0}$, $\pi_{1,0}$, and $\pi_{1,1}$. Again from (10), $\Pi_{1}(z)$ is a function of $\Pi_{0}(z)$, $\pi_{0,0}, \pi_{1,0}$, and $\pi_{1,1}$. Thus, if $\pi_{0,0}, \pi_{1,0}$, and $\pi_{1,1}$ are found out, then $\Pi_{0}(z)$ and $\Pi_{1}(z)$ are completely computed. In the next subsection, we find the probabilities $\pi_{0,0}, \pi_{1,0}$, and $\pi_{1,1}$ and the expected system sizes.

3.2. Mean System Sizes. Let us suppose that $E\left[L_{0}\right]$ and $E\left[L_{1}\right]$ are the expected number of customers in the system when the server is on a working vacation period and in a normal busy period, respectively.

Adding (4) and (5) over all possible values of $n$, we get

$$
(\phi+\bar{q} \eta) \Pi_{0}(1)=\mu \pi_{1,1}+(\phi+\bar{q} \eta) \pi_{0,0}+\bar{q} \eta \pi_{1,0} .
$$

Using (9), we get

$$
A_{1}=(\phi+\bar{q} \eta) \Pi_{0}(1)
$$

Now (10) can be rewritten as

$$
\Pi_{1}(z)=\frac{(\phi z+\bar{q} \eta) \Pi_{0}(z)-z(\phi+\bar{q} \eta) \Pi_{0}(1)}{(\lambda z-\mu)(1-z)}-\frac{\bar{q} \eta \pi_{0,0}}{\lambda z-\mu} .
$$

Using L'Hôpital's rule, we find

$$
\Pi_{1}(1)=\left(\frac{\phi+\bar{q} \eta}{\mu-\lambda}\right) \Pi_{0}^{\prime}(1)+\frac{\bar{q} \eta}{\mu-\lambda}\left(\pi_{0,0}-\Pi_{0}(1)\right)
$$

where $\Pi_{0}^{\prime}(1)=E\left[L_{0}\right]$, that is, the expected number of customers in the system when the server is on a working vacation period. Since $\Pi_{1}(1)=1-\Pi_{0}(1)$, we get from (22)

$$
\begin{aligned}
E\left[L_{0}\right]= & \left(\frac{\mu-\lambda}{\phi+\bar{q} \eta}\right)\left(1-\Pi_{0}(1)\right) \\
& -\left(\frac{\bar{q} \eta}{\phi+\bar{q} \eta}\right)\left(\pi_{0,0}-\Pi_{0}(1)\right) .
\end{aligned}
$$


We need to deduce the proportion of time the server is on a working vacation period $\left(\Pi_{0}(1)\right)$, so that $E\left[L_{0}\right]$ can be obtained. Adding (3)-(5) and rearranging the terms, we obtain

$$
\begin{aligned}
& \lambda \pi_{n, 0}+\lambda \pi_{n, 1}-\left[(\eta+(n+1) \xi) \pi_{n+1,0}+\mu \pi_{n+1,1}\right] \\
& \quad=\lambda \pi_{n-1,0}+\lambda \pi_{n-1,1}-\left[(\eta+n \xi) \pi_{n, 0}+\mu \pi_{n, 1}\right], \quad n \geq 1 .
\end{aligned}
$$

Using recursively (24) and applying (2), we get

$$
\lambda \pi_{n, 0}+\lambda \pi_{n, 1}=(\eta+(n+1) \xi) \pi_{n+1,0}+\mu \pi_{n+1,1}, \quad n \geq 0 .
$$

Adding over all possible values of $n$ in (25), we obtain

$$
\begin{aligned}
\lambda \Pi_{0}(1)+\lambda \Pi_{1}(1)= & \mu \Pi_{1}(1)+\eta\left(\Pi_{0}(1)-\pi_{0,0}\right) \\
& +\xi \sum_{n=0}^{\infty}(n+1) \pi_{n+1,0}, \quad n \geq 0 .
\end{aligned}
$$

Note that $E\left[L_{0}\right]=\sum_{n=0}^{\infty}(n+1) \pi_{n+1,0}$ and $\Pi_{1}(1)=1-\Pi_{0}(1)$. By substituting the value of $E\left[L_{0}\right]$ from (23) in (26) and solving we get

$$
\begin{aligned}
(\xi+ & \phi+\bar{q} \eta)(\mu-\lambda) \\
= & {[\xi(\mu-\lambda)+(\mu-\eta)(\phi+\bar{q} \eta)-\xi \bar{q} \eta] \Pi_{0}(1) } \\
& +\{\xi \bar{q} \eta+\eta(\phi+\bar{q} \eta)\} \pi_{0,0} .
\end{aligned}
$$

Taking limit $z \rightarrow 1$ in (14) and using (9) and (20), we get

$$
\begin{aligned}
\Pi_{0}(1)=\frac{e^{\lambda / \xi}}{\xi}[ & -(\phi+\bar{q} \eta) \Pi_{0}(1) K_{1}(1) \\
& \left.+q \eta \pi_{0,0} K_{2}(1)\right] \lim _{z \rightarrow 1}(1-z)^{-(\phi+\bar{q} \eta) / \xi} .
\end{aligned}
$$

As $0 \leq \Pi_{0}(1)=\sum_{n=0}^{\infty} \pi_{n, 0} \leq 1$ and $\lim _{z \rightarrow 1}(1-z)^{-(\phi+\bar{q} \eta) / \xi} \rightarrow$ $\infty$, so we must have

$$
-(\phi+\bar{q} \eta) \Pi_{0}(1) K_{1}(1)+q \eta \pi_{0,0} K_{2}(1)=0 .
$$

Using (27) and (29), we get

$$
\begin{aligned}
& \pi_{0,0}=\frac{(\phi+\bar{q} \eta) K_{1}(1)}{q \eta K_{2}(1)} \Pi_{0}(1), \\
& \Pi_{0}(1)=\left((\xi+\phi+\bar{q} \eta)(\mu-\lambda) q K_{2}(1)\right) \\
& \times\left(\{\xi \bar{q}+\phi+\bar{q} \eta\}(\phi+\bar{q} \eta) K_{1}(1)\right. \\
&+[\xi(\mu-\lambda)+(\mu-\eta)(\phi+\bar{q} \eta)-\xi \bar{q} \eta] \\
&\left.\times q K_{2}(1)\right)^{-1} .
\end{aligned}
$$

Thus $E\left[L_{0}\right]$ is found from (23). Using (2) and (20) the unknowns $\pi_{1,0}$ and $\pi_{1,1}$ are obtained as follows:

$$
\begin{gathered}
\pi_{1,0}=\frac{(\phi+\bar{q} \eta)\left\{(\lambda+\phi+\bar{q} \eta) K_{1}(1)-q \eta K_{2}(1)\right\}}{(\xi+q \eta) q \eta K_{2}(1)} \Pi_{0}(1), \\
\pi_{1,1}=\left(\left(( \phi + \overline { q } \eta ) \left\{(\xi+\eta) q \eta K_{2}(1)\right.\right.\right. \\
\left.\left.-K_{1}(1)(\lambda \bar{q} \eta+(\xi+\eta)(\phi+\bar{q} \eta))\right\}\right) \\
\left.\times\left(\mu q \eta K_{2}(1)(\xi+q \eta)\right)^{-1}\right) \Pi_{0}(1) .
\end{gathered}
$$

Now, the stationary probabilities $\pi_{n, 0}$ and $\pi_{n, 1}$ can be derived by using (3)-(5) in terms of $\pi_{0,0}, \pi_{1,0}$, and $\pi_{1,1}$.

The expected number of customers in the system when the server is in a normal busy period $\left(E\left[L_{1}\right]\right)$ can be obtained from (21) by using L'Hôpital's rule as follows:

$$
\begin{aligned}
E\left[L_{1}\right]= & \Pi_{1}^{\prime}(1)=\frac{\phi+\bar{q} \eta}{(\mu-\lambda)} \frac{\Pi_{0}^{\prime \prime}(1)}{2}+\frac{1}{(\phi+\bar{q} \eta)(\mu-\lambda)} \\
& \times\left[(\phi \mu+\lambda \bar{q} \eta)\left(1-\Pi_{0}(1)\right)+\phi \bar{q} \eta\left(\Pi_{0}(1)-\pi_{0,0}\right)\right] .
\end{aligned}
$$

Differentiating (7) twice at $z=1$, we obtain

$$
\begin{aligned}
& g^{\prime \prime}(1) \Pi_{0}(1)+2\left(g^{\prime}(1)-\xi\right) \Pi_{0}^{\prime}(1) \\
& \quad+(g(1)-2 \xi) \Pi_{0}^{\prime \prime}(1)=0
\end{aligned}
$$

It is easily examined that $g(1)=-(\phi+\bar{q} \eta), g^{\prime}(1)=\lambda-(\eta+\phi)$, and $g^{\prime \prime}(1)=2 \lambda$. Thus, from (33), we get

$$
\frac{1}{2} \Pi_{0}^{\prime \prime}(1)=\left(\frac{\lambda}{\phi+\bar{q} \eta+2 \xi}\right) \Pi_{0}(1)-\left(\frac{\xi+\eta+\phi-\lambda}{\phi+\bar{q} \eta+2 \xi}\right) E\left[L_{0}\right] .
$$

Using (34) and (23) in (32), we get $E\left[L_{1}\right]$.

The expected number of customers in the system can be computed as $E[L]=E\left[L_{0}\right]+E\left[L_{1}\right]$.

3.3. Sojourn Times. Let $W$ be the total sojourn time of a customer in the system, evaluated from the instant of arrival till departure, with the departure either due to completion of service or as a consequence of abandonment. We have by Little's rule

$$
E[W]=\frac{1}{\lambda}\left(E\left[L_{0}\right]+E\left[L_{1}\right]\right) .
$$

Let $W_{\text {ser }}$ be defined as the total sojourn time of a customer who completes his service before departing the system. Let $W_{n, j}$ be the conditional sojourn time of a customer who does not abandon the system, given that the state upon his arrival is $(n, j)$. Then, we have

$$
E\left[W_{n, 1}\right]=\frac{n+1}{\mu}, \quad n=1,2, \ldots
$$


Let $P_{(n, 0)(n, 1)}$ be the transition probability from state $(n, 0)$ to state $(n, 1)$, let $P_{(n, 0)(n+1,0)}$ be the transition probability from state $(n, 0)$ to state $(n+1,0)$, let $P_{(n+1,0)(n, 1)}$ be the transition probability from state $(n+1,0)$ to state $(n, 1)$, and let $P_{(n, 0)(n-1,0)}$ be the transition probability from state $(n, 0)$ to state $(n-1,0)$. We compute $E\left[W_{n 0}\right]$ by using the approach of Altman and Yechiali [12]. For $n \geq 1$, we get

$$
\begin{aligned}
E\left[W_{n, 0}\right]= & P_{(n+1,0)(n+1,1)}\left(\frac{1}{\alpha_{n+1}}+E\left[W_{n, 1}\right]\right) \\
& +P_{(n+1,0)(n+2,0)}\left(\frac{1}{\alpha_{n+1}}+E\left[W_{n, 0}\right]\right) \\
& +P_{(n+1,0)(n, 0)}\left(\frac{1}{\alpha_{n+1}}+E\left[W_{n-1,0}\right]\right) \\
& +P_{(n+1,0)(n, 1)}\left(\frac{1}{\alpha_{n+1}}+E\left[W_{n-1,1}\right]\right)
\end{aligned}
$$

where $\alpha_{n}=\lambda+\phi+\eta+n \xi$ for $n=0,1,2, \ldots$. The mean sojourn time of the marked customer in the state $(n+1,0)$ for transition to another state is $1 / \alpha_{n+1}$.

The second term above follows because a new arrival does not change the sojourn time of a customer present in the system. In the third term, $n$ customers can leave the system as our customer is not impatient and the fourth term follows due to vacation interruption. The transition probabilities in (37) are given as

$$
\begin{gathered}
P_{(n+1,0)(n+1,1)}=\frac{\phi}{\alpha_{n+1}}, \quad P_{(n+1,0)(n, 1)}=\frac{\bar{q} \eta}{\alpha_{n+1}}, \\
P_{(n+1,0)(n+2,0)}=\frac{\lambda}{\alpha_{n+1}}, \\
P_{(n+1,0)(n, 0)}=\frac{(n+1) \xi}{\alpha_{n+1}}\left(\frac{n}{n+1}\right)+\frac{q \eta}{\alpha_{n+1}}=\frac{n \xi+q \eta}{\alpha_{n+1}},
\end{gathered}
$$

for $n \geq 0$.

Thus, $E\left[W_{n 0}\right]$ is given as

$$
\begin{aligned}
E\left[W_{n, 0}\right]= & \frac{\phi}{\alpha_{n+1}}\left(\frac{1}{\alpha_{n+1}}+E\left[W_{n, 1}\right]\right) \\
& +\frac{\lambda}{\alpha_{n+1}}\left(\frac{1}{\alpha_{n+1}}+E\left[W_{n, 0}\right]\right) \\
& +\frac{\bar{q} \eta}{\alpha_{n+1}}\left(\frac{1}{\alpha_{n+1}}+E\left[W_{n-1,1}\right]\right) \\
& +\frac{n \xi+q \eta}{\alpha_{n+1}}\left(\frac{1}{\alpha_{n+1}}+E\left[W_{n-1,0}\right]\right) .
\end{aligned}
$$

For $n=0$, we get

$$
E\left[W_{0,0}\right]=\frac{1}{\phi+\eta+\xi}\left(\frac{\alpha_{0}}{\alpha_{1}}+\frac{\phi}{\mu}\right) .
$$

Recursively iterating (39) for $n \geq 0$ and using (40), we get

$$
\begin{aligned}
E\left[W_{n, 0}\right]= & \frac{1}{\phi+\eta+(n+1) \xi}\left[\frac{\alpha_{n}}{\alpha_{n+1}}+\frac{(n+1) \phi+n \bar{q} \eta}{\mu}\right] \\
& +\sum_{i=1}^{n} \frac{1}{\phi+\eta+i \xi}\left(\frac{\alpha_{i-1}}{\alpha_{i}}+\frac{i \phi+(i-1) \bar{q} \eta}{\mu}\right) \\
& \times \prod_{j=i}^{n}\left(\frac{q \eta+j \xi}{\phi+\eta+(j+1) \xi}\right) .
\end{aligned}
$$

The mean waiting time of customers served by the system is given by

$$
\begin{aligned}
E\left[W_{\text {ser }}\right] & =\sum_{n=0}^{\infty} \pi_{n, 0} E\left[W_{n, 0}\right]+\sum_{n=1}^{\infty} \pi_{n, 1} E\left[W_{n, 1}\right] \\
& =\sum_{n=0}^{\infty} \pi_{n, 0} E\left[W_{n, 0}\right]+\frac{1}{\mu}\left(E\left[L_{1}\right]+\Pi_{1}(1)\right) .
\end{aligned}
$$

3.3.1. Performance Measures. The other two important performance measures are the proportion of customers served, denoted by $P_{s}$, and the rate of abandonment due to impatience, denoted by $R$. The expected number of customers served per unit of time is $\mu \Pi_{1}(1)+\eta\left(\Pi_{0}(1)-\pi_{0,0}\right)$ signifying that the proportion of customers served is

$$
P_{s}=\frac{1}{\lambda}\left\{\mu \Pi_{1}(1)+\eta\left(\Pi_{0}(1)-\pi_{0,0}\right)\right\} .
$$

The rate of abandonment $(R)$ of a customer due to impatience is given by

$$
R=\xi E\left[L_{0}\right]=\lambda-\left\{\mu \Pi_{1}(1)+\eta\left(\Pi_{0}(1)-\pi_{0,0}\right)\right\},
$$

which follows from (26). The probability that the system is in normal busy period $\left(P_{b}\right)$ and the probability that the system is in working vacation $\left(P_{\mathrm{wv}}\right)$ are, respectively, given by

$$
P_{b}=\sum_{n=1}^{\infty} \pi_{n, 1}=\Pi_{1}(1), \quad P_{\mathrm{wv}}=\sum_{n=0}^{\infty} \pi_{n, 0}=\Pi_{0}(1) .
$$

\section{Stochastic Decomposition Results}

We experience that the working vacation queues have the stochastic decomposition structures for queue length and waiting times. In order to have a preferable comparison with the existing models in many instances it is of concern to decompose the quantities of interest into various factors. The stationary queue length (or stationary waiting time) in the impatient Bernoulli schedule vacation interruption model discussed in this paper can also be decomposed into sum of two independent random variables which are the corresponding stationary queue length (or stationary waiting time) in the classical $M / M / 1$ queue without vacation and additional queue length (or additional waiting time) due to the impatient Bernoulli schedule vacation interruption effect. 
Theorem 4. If $\rho<1$, the stationary queue length $L$ can be decomposed into the sum of two independent random variables: $L=L_{c}+L_{d}$, where $L_{c}$ is the stationary queue length of a classical $M / M / 1$ queue without vacations and the additional stationary queue length $L_{d}$ due to the Bernoulli schedule vacation interruption has a distribution with its pgf:

$$
\begin{aligned}
L_{d}(z)=\frac{1}{1-\rho}[ & \Pi_{0}(z)\left(1-\rho z-\frac{\phi z+\bar{q} \eta}{\mu(1-z)}\right) \\
& \left.+\frac{z(\phi+\bar{q} \eta) \Pi_{0}(1)}{\mu(1-z)}+\frac{\bar{q} \eta \pi_{0,0}}{\mu}\right] .
\end{aligned}
$$

Proof. Consider

$$
\begin{aligned}
L(z)= & \Pi_{0}(z)+\Pi_{1}(z) \\
= & \Pi_{0}(z)\left(1+\frac{\phi z+\bar{q} \eta}{(\lambda z-\mu)(1-z)}\right) \\
& -\frac{z(\phi+\bar{q} \eta)}{(\lambda z-\mu)(1-z)} \Pi_{0}(1)-\frac{\bar{q} \eta \pi_{0,0}}{\lambda z-\mu} \\
= & \left(\frac{\mu-\lambda}{\mu-\lambda z}\right)\left[\Pi_{0}(z)\left(\frac{\mu-\lambda z}{\mu-\lambda}-\frac{\phi z+\bar{q} \eta}{(\mu-\lambda)(1-z)}\right)\right. \\
& \left.\quad+\frac{\bar{q} \eta \pi_{0,0}}{\mu-\lambda}+\frac{z(\phi+\bar{q} \eta) \Pi_{0}(1)}{(\mu-\lambda)(1-z)}\right] \\
= & \left(\frac{1-\rho}{1-\rho z}\right) \times L_{d}(z),
\end{aligned}
$$

where $L_{d}(z)$ is the pgf of the additional queue length due to the Bernoulli schedule vacation interruption and is given by

$$
\begin{aligned}
& L_{d}(z)=\frac{1}{1-\rho}[ \Pi_{0}(z)\left((1-\rho z)-\frac{\phi z+\bar{q} \eta}{\mu(1-z)}\right) \\
&\left.+\frac{z(\phi+\bar{q} \eta) \Pi_{0}(1)}{\mu(1-z)}+\frac{\bar{q} \eta \pi_{0,0}}{\mu}\right] \\
&=\frac{1}{1-\rho}\left[\sum_{n=0}^{\infty} \pi_{n, 0} z^{n}-\rho \sum_{n=0}^{\infty} \pi_{n, 0} z^{n+1}\right.\left.\quad+\frac{\phi}{\mu} \sum_{n=1}^{\infty} \sum_{j=0}^{\infty} \pi_{n+j, 0} z^{n}+\frac{\bar{q} \eta}{\mu} \sum_{n=1}^{\infty} \sum_{j=0}^{\infty} \pi_{n+j+1,0} z^{n}\right] \\
&=\sum_{n=0}^{\infty} t_{n} z^{n},
\end{aligned}
$$

where $t_{0}=\pi_{0,0} /(1-\rho)$ and $t_{n}=(1 /(1-\rho))\left[\pi_{n, 0}-\rho \pi_{n-1,0}+\right.$ $\left.(\phi / \mu) \sum_{j=0}^{\infty} \pi_{n+j, 0}+(\bar{q} \eta / \mu) \sum_{j=0}^{\infty} \pi_{n+j+1,0}\right], n \geq 1$.
Now, we show that $\sum_{n=0}^{\infty} t_{n}=1$ for $t_{n} \in[0,1]$ :

$$
\begin{aligned}
& \sum_{n=0}^{\infty} t_{n}=\frac{1}{1-\rho}\left[\sum_{n=0}^{\infty} \pi_{n, 0}-\rho \sum_{n=1}^{\infty} \pi_{n-1,0}+\frac{\phi}{\mu} \sum_{n=1}^{\infty} \sum_{j=0}^{\infty} \pi_{n+j, 0}\right. \\
& \left.+\frac{\bar{q} \eta}{\mu} \sum_{n=1}^{\infty} \sum_{j=0}^{\infty} \pi_{n+j+1,0}\right] \\
& =\frac{1}{1-\rho}\left[(1-\rho) \sum_{n=0}^{\infty} \pi_{n, 0}+\frac{\phi}{\mu} \sum_{n=1}^{\infty} n \pi_{n, 0}\right. \\
& \left.+\frac{\bar{q} \eta}{\mu} \sum_{n=2}^{\infty}(n-1) \pi_{n, 0}\right] \\
& =\frac{1}{1-\rho}\left[(1-\rho) \sum_{n=0}^{\infty} \pi_{n, 0}+\frac{\phi+\bar{q} \eta}{\mu} \sum_{n=1}^{\infty} n \pi_{n, 0}\right. \\
& \left.-\frac{\bar{q} \eta}{\mu} \sum_{n=2}^{\infty} \pi_{n, 0}\right] \text {. }
\end{aligned}
$$

Using (23), we get

$$
\begin{aligned}
\sum_{n=0}^{\infty} t_{n}= & \sum_{n=0}^{\infty} \pi_{n, 0}+\frac{1}{1-\rho} \\
& \times\left[(1-\rho)\left(1-\Pi_{0}(1)\right)-\frac{\bar{q} \eta}{\mu}\left(\pi_{0,0}-\Pi_{0}(1)\right)\right. \\
& \left.-\frac{\bar{q} \eta}{\mu} \sum_{n=2}^{\infty} \pi_{n, 0}\right] \\
= & \sum_{n=0}^{\infty} \pi_{n, 0}+1-\Pi_{0}(1)+\frac{\bar{q} \eta}{\mu(1-\rho)} \Pi_{0}(1) \\
& -\frac{\bar{q} \eta}{\mu(1-\rho)} \sum_{n=0}^{\infty} \pi_{n, 0}=1 .
\end{aligned}
$$

Thus, $L_{d}(z)$ is a pgf of the additional queue length due to the Bernoulli schedule vacation interruption.

Theorem 5. If $\rho<1$, the stationary waiting time $W$ can be decomposed into the sum of two independent random variables: $W=W_{c}+W_{d}$, where $W_{c}$ is the stationary waiting time of a $M / M / 1$ queue without vacations, which has an exponential distribution with the parameter $\mu(1-\rho)$, and the additional stationary waiting time $W_{d}$ due to the Bernoulli schedule vacation interruption has a distribution with its Laplace Stieltjes transform (LST):

$$
\begin{aligned}
W_{d}^{*}(s)= & \frac{1}{(\mu-\lambda) s} \\
& \times[\{(\mu-\lambda+s) s-\phi(\lambda-s)-\lambda \bar{q} \eta\} \\
& \quad \times \Pi_{0}\left(1-\frac{s}{\lambda}\right)+(\lambda-s)(\phi+\bar{q} \eta) \Pi_{0}(1) \\
& \left.+\bar{q} \eta s \pi_{0,0}\right] .
\end{aligned}
$$


TABLE 1: Impact of $q$ and $\xi$ on some performance measures.

\begin{tabular}{|c|c|c|c|c|c|c|c|}
\hline$q$ & $\xi$ & $E\left[L_{0}\right]$ & $E\left[L_{1}\right]$ & $E[L]$ & $E\left[W_{\text {ser }}\right]$ & $P_{s}$ & $R$ \\
\hline \multirow{3}{*}{0.2} & 0.5 & 0.50781 & 1.42817 & 1.93598 & 0.68714 & 0.91537 & 0.25390 \\
\hline & 1.0 & 0.47609 & 1.20609 & 1.68218 & 0.59041 & 0.84130 & 0.47609 \\
\hline & 1.5 & 0.44683 & 1.04633 & 1.49315 & 0.51786 & 0.77659 & 0.67024 \\
\hline \multirow{3}{*}{0.5} & 0.5 & 0.57282 & 1.40210 & 1.97492 & 0.68261 & 0.90453 & 0.28641 \\
\hline & 1.0 & 0.52567 & 1.16015 & 1.68582 & 0.57377 & 0.82478 & 0.52567 \\
\hline & 1.5 & 0.48521 & 0.99527 & 1.48048 & 0.49603 & 0.75740 & 0.72781 \\
\hline \multirow{3}{*}{0.8} & 0.5 & 0.66660 & 1.36103 & 2.02763 & 0.67758 & 0.88890 & 0.33330 \\
\hline & 1.0 & 0.59161 & 1.09745 & 1.68907 & 0.55277 & 0.80280 & 0.59161 \\
\hline & 1.5 & 0.53339 & 0.93042 & 1.46381 & 0.46949 & 0.73330 & 0.80009 \\
\hline \multirow{3}{*}{1.0} & 0.5 & 0.75748 & 1.31811 & 2.07559 & 0.67367 & 0.87375 & 0.37874 \\
\hline & 1.0 & 0.64949 & 1.04124 & 1.69073 & 0.53508 & 0.78350 & 0.64949 \\
\hline & 1.5 & 0.57311 & 0.87647 & 1.44957 & 0.44815 & 0.71345 & 0.85966 \\
\hline
\end{tabular}

TABLE 2: Impact of $q$ and $\eta$ on some performance measures.

\begin{tabular}{|c|c|c|c|c|c|c|c|}
\hline$q$ & $\eta$ & $E\left[L_{0}\right]$ & $E\left[L_{1}\right]$ & $E[L]$ & $E\left[W_{\text {ser }}\right]$ & $P_{s}$ & $R$ \\
\hline \multirow{3}{*}{0.2} & 1.0 & 1.19062 & 2.39321 & 3.58384 & 0.91303 & 0.85117 & 0.59531 \\
\hline & 2.0 & 0.79209 & 2.07999 & 2.87208 & 0.75737 & 0.90099 & 0.39604 \\
\hline & 3.0 & 0.60909 & 1.84852 & 2.45761 & 0.66606 & 0.92386 & 0.30455 \\
\hline \multirow{3}{*}{0.5} & 1.0 & 1.45114 & 2.34523 & 3.79637 & 0.95209 & 0.81861 & 0.72557 \\
\hline & 2.0 & 0.99371 & 2.01627 & 3.00998 & 0.77719 & 0.87579 & 0.49686 \\
\hline & 3.0 & 0.76495 & 1.75810 & 2.52305 & 0.66704 & 0.90438 & 0.38247 \\
\hline \multirow{3}{*}{0.8} & 1.0 & 1.90323 & 2.21990 & 4.12313 & 1.01149 & 0.76210 & 0.95162 \\
\hline & 2.0 & 1.39782 & 1.84657 & 3.24440 & 0.81125 & 0.82527 & 0.69891 \\
\hline & 3.0 & 1.08832 & 1.54154 & 2.62985 & 0.66846 & 0.86396 & 0.54416 \\
\hline \multirow{3}{*}{1.0} & 1.0 & 2.45678 & 2.02065 & 4.47743 & 1.07461 & 0.69290 & 1.22839 \\
\hline & 2.0 & 2.03215 & 1.51669 & 3.54884 & 0.85407 & 0.74598 & 1.01607 \\
\hline & 3.0 & 1.63857 & 1.12327 & 2.76184 & 0.66636 & 0.79518 & 0.81929 \\
\hline
\end{tabular}

Proof. The stationary queue length and waiting time of a customer distribution form of Little's law, as in Keilson and Servi [18], have the relation

$$
L(z)=W^{*}(\lambda(1-z)) .
$$

Let us assume $s=\lambda(1-z)$, so $z=(1-s / \lambda)$ and $1-z=s / \lambda$. Setting these relations in (46), we obtain the required result. Taking $\lim _{s \rightarrow 0} W_{d}^{*}(s)$, we obtain

$$
\begin{aligned}
W_{d}= & \frac{1}{\mu-\lambda} \\
& \times\left[(\mu-\lambda+\phi) \Pi_{0}(1)+(\phi+\bar{q} \eta) \Pi_{0}^{\prime}(1)\right. \\
& \left.\quad-(\phi+\bar{q} \eta) \Pi_{0}(1)+\bar{q} \eta \pi_{0,0}\right] .
\end{aligned}
$$

Substituting the value of $\Pi_{0}^{\prime}(1)$ from (23), we get after simplification $\lim _{s \rightarrow 0} W_{d}^{*}(s)=1$. This proves that $W_{d}$ is a random variable of the additional waiting time.

\section{Numerical Results}

In this section, we give some numerical results to study the various performance measures. Tables 1 and 2 present the numerical results of the various performance measures such as the expected number of customers when the system is on working vacation $\left(E\left[L_{0}\right]\right)$, the expected number of customers when the system is on busy period $\left(E\left[L_{1}\right]\right)$, the expected number of customers in the system $(E[L])$, the expected waiting time of customers served by the system $\left(E\left[W_{\text {ser }}\right]\right)$, the proportion of customers served $\left(P_{s}\right)$, and the rate of abandonment $(R)$ of a customer due to impatience by varying $q$, $\xi$, and $\eta$. The parameters for Tables 1 and 2 are taken as $\lambda=3.0, \mu=5.0, \phi=1.0, \eta=2.0$ and $\lambda=4.0, \mu=6.0$, $\phi=0.3, \xi=0.5$, respectively. From Table 1 , we observe that, for fixed $\xi, E\left[L_{0}\right], E[L]$, and $R$ increase as $q$ increases. Further, $E\left[L_{1}\right], E\left[W_{\text {ser }}\right]$, and $P_{s}$ decrease as $q$ increases. For fixed $q$, the expected number of customers in the system $E\left[L_{0}\right]$, $E\left[L_{1}\right], E[L], E\left[W_{\text {ser }}\right]$, and $P_{s}$ decreases as $\xi$ increases. But the rate of abandonment $(R)$ of a customer due to impatience increases as $\xi$ increases. From Table 2, it can be seen that, for fixed $\eta, E\left[L_{0}\right], E[L], E\left[W_{\text {ser }}\right]$, and $R$ increase as probability $q$ increases. However, for fixed probability $q, E\left[L_{0}\right], E\left[L_{1}\right]$, $E[L], E\left[W_{\text {ser }}\right]$, and $R$ follow a decreasing trend as $\eta$ increases. But the proportion of customers served $\left(P_{s}\right)$ increases with the increase of $\eta$. As expected, we observe that the model with small values of Bernoulli schedule parameter $q$ performs better. 


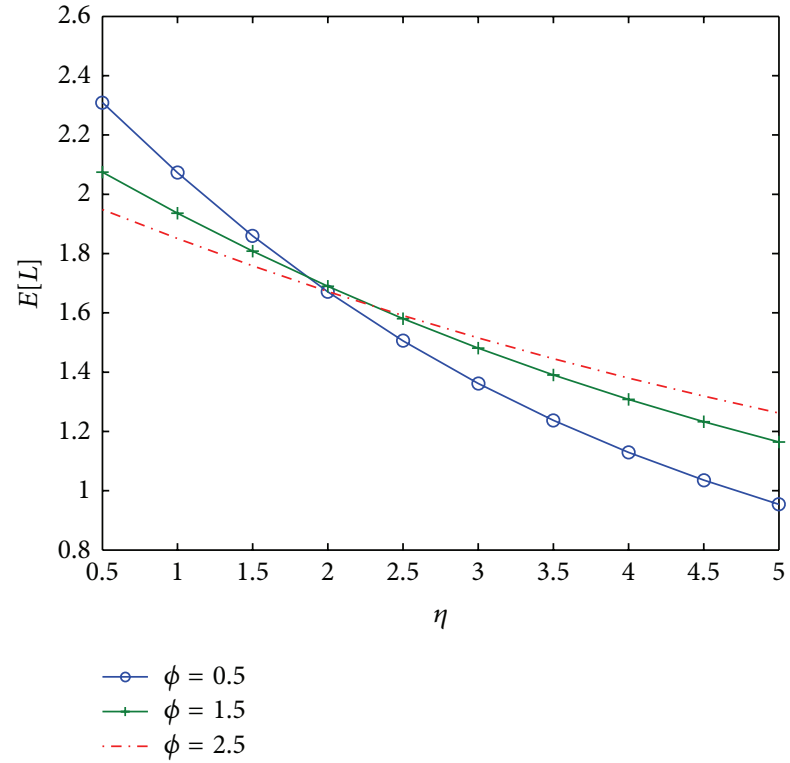

Figure 1: Effect of $\eta$ on $E[L]$.

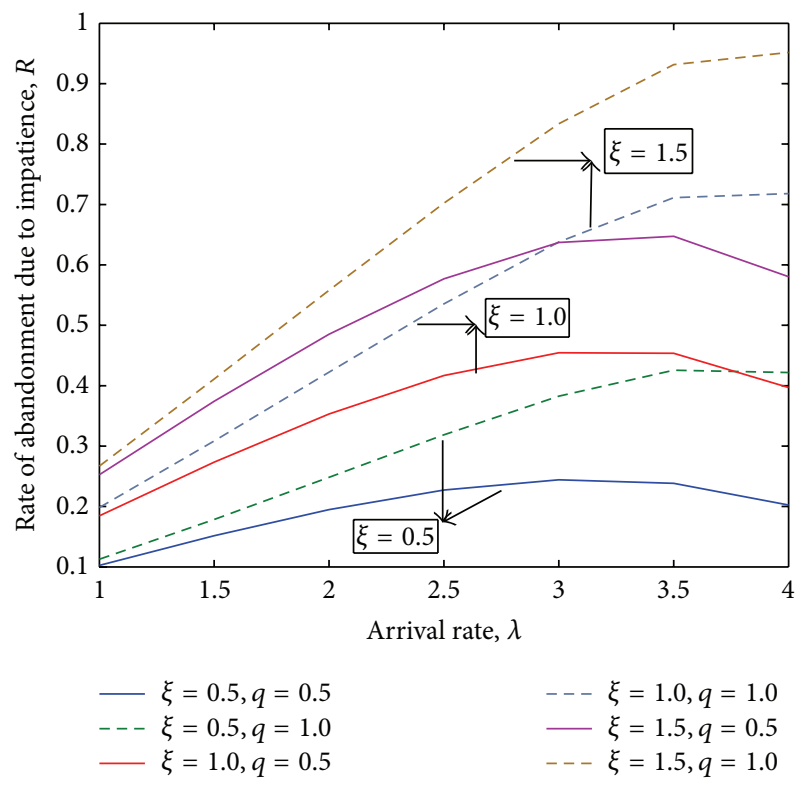

Figure 2: Effect of $\lambda$ on $R$.

The impact of service rate during vacation $(\eta)$ on the expected number of customers in the system $(E[L])$ for various vacation rates $(\phi)$ is shown in Figure 1. The parameters are taken as $\lambda=3.0, \mu=5.0 ; \xi=1.0$ and $q=1.0$. We observe that as service rate during vacation increases, the expected number of customers in the system decreases. When $\eta>2$, $E[L]$ increases with the increase of vacation rate $(\phi)$, but when $\eta<2, E[L]$ decreases with the increase of vacation rate. Hence, a desirable performance can be obtained by choosing the proper value of $\phi$, which is consistent with the view that increase of the vacation rates may increase the queue length.

Figure 2 plots the effect of arrival rate $(\lambda)$ on the rate of abandonment $(R)$ of a customer due to impatience for

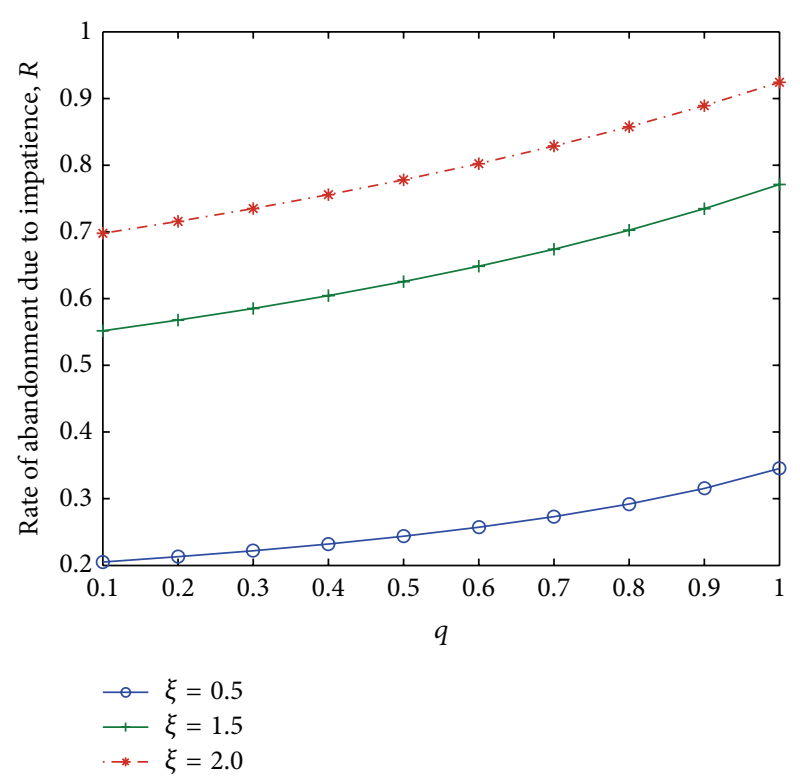

Figure 3: Effect of $q$ on $R$.

various parameters $\xi$ and $q$. The parameters are taken as $\mu=5.0, \phi=0.5$, and $\eta=4.0$. It may be seen that the rate of abandonment $(R)$ of a customer due to impatience first increases and then diminishes with increasing the arrival rate for both $q=0.5$ and $q=1.0$. As expected, the rate of abandonment of a customer monotonically increases as $\xi$ increases because more numbers of customers renege and leave the system. Furthermore, Bernoulli schedule vacation interruption model outperforms the multiple working vacations model.

Figure 3 illustrates the impact of probability $q$ on the rate of abandonment $(R)$ of a customer for various impatient timers $\xi$. The parameters are taken as $\lambda=3.0, \mu=7.0$, $\phi=0.5$, and $\eta=5.0$. It can be seen that as the vacation interruption probability $q$ increases, the rate of abandonment $(R)$ of a customer due to impatience also increases. But, with the increase of parameter $\xi$, the rate of abandonment of a customer due to impatience increases.

Figure 4 shows dependence of the proportion of customers served $\left(P_{s}\right)$ on the $\phi$ and $\xi$. The parameters are $\lambda=$ 3.0, $\mu=5.0, \eta=3.0$, and $q=0.5$. It is observed that, for fixed $\phi$, the proportion of customers served decreases when $\xi$ increases. Further with fixed $\xi$ the proportion of customers served increases when $\phi$ increases. Therefore, we can set an acceptable region in terms of the $\phi$ and $\xi$ so that the proportion of customers served can be maximized. Figure 5 compares the impact of $\xi$ on the expected waiting time of customers served by the system $\left(E\left[W_{\text {ser }}\right]\right)$. The parameters are $\lambda=3.0, \mu=5.0, \eta=3.0$, and $\phi=1.5$. It is evident from the figure that, for fixed probability $q$, as $\xi$ increases the expected waiting time of customers served by the system decreases. But the expected waiting time of customers served by the system is higher for $q=0$, that is, the vacation interruption model. As we can see, with the variation of probability $q$ there is a significant impact in the expected waiting time of customers served by the system. 


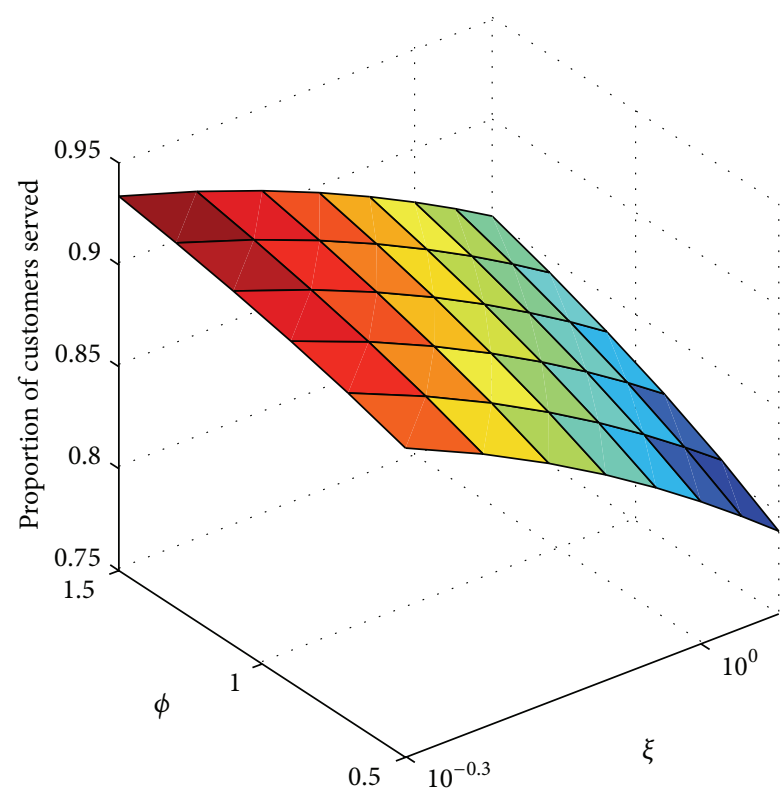

Figure 4: Effect of $\phi$ and $\xi$ on $P_{s}$.

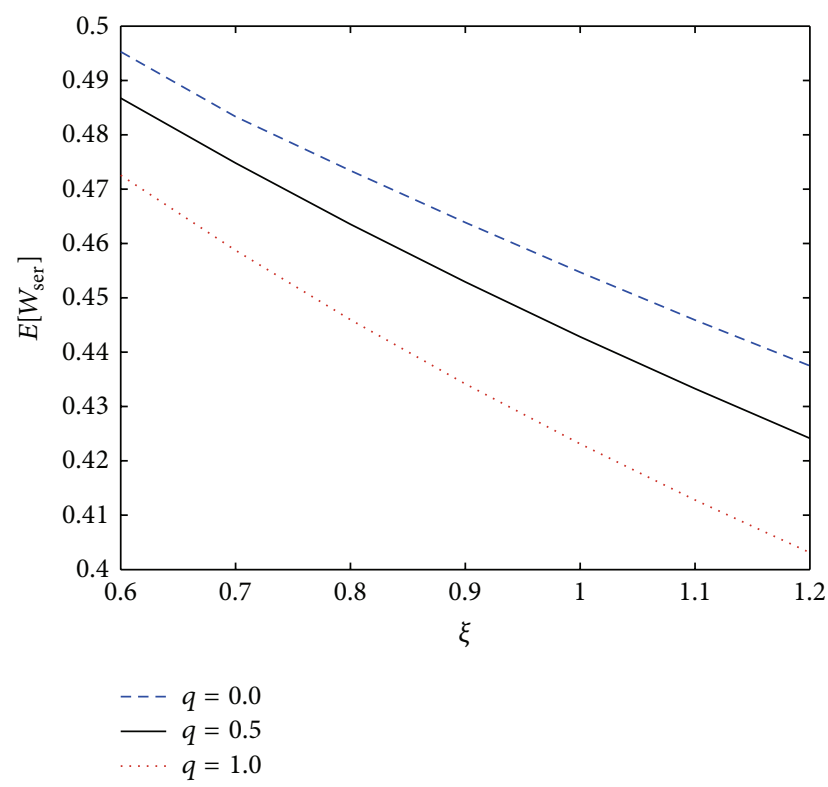

Figure 5: Impact of $\xi$ on $E\left[W_{\text {ser }}\right]$.

\section{Conclusions}

In this paper, we have carried out the impact of impatient behavior of customers in $M / M / 1$ multiple working vacations queue with Bernoulli schedule vacation interruptions, where the customer's impatience is because of slow service rate in a working vacation. We have obtained the explicit expressions for the probability generating functions of the number of customers in the system, when the system is in a vacation interruption period and in a normal busy period, respectively. Various performance measures such as the mean number of customers in the system when the server was in a service, the mean sojourn time of a customer served, the proportion of customers served, and the rate of abandonment due to impatience are evaluated. The impacts of some parameters on the performance measures of the system have been investigated numerically.

\section{Conflict of Interests}

The author declares that there is no conflict of interests regarding the publication of this paper.

\section{References}

[1] J. C. Ke, C. H. Wu, and Z. G. Zhang, "Recent developments in vacation queueing models: a short survey," International Journal of Operations Research, vol. 7, no. 4, pp. 3-8, 2010.

[2] N. Tian and Z. G. Zhang, Vacation Queueing Models, Springer, 2006.

[3] D. Yue, Y. Zhang, and W. Yue, "Optimal performance analysis of an $M / M / 1 / N$ queue system with balking, reneging and server vacation," International Journal of Pure and Applied Mathematics, vol. 28, no. 1, pp. 101-115, 2006.

[4] L. D. Servi and S. G. Finn, " $M / M / 1$ queues with working vacations $(M / M / 1 / W V)$," Performance Evaluation, vol. 50, no. 1, pp. 41-52, 2002.

[5] W. Y. Liu, X. L. Xu, and N. S. Tian, "Stochastic decompositions in the $M / M / 1$ queue with working vacations," Operations Research Letters, vol. 35, no. 5, pp. 595-600, 2007.

[6] Y. Baba, "The M/PH/1 queue with working vacations and vacation interruption," Journal of Systems Science and Systems Engineering, vol. 19, no. 4, pp. 496-503, 2010.

[7] H. Chen, J. Li, and N. Tian, "The GI/M/I queue with phasetype working vacations and vacation interruption," Journal of Applied Mathematics and Computing, vol. 30, no. 1-2, pp. 121141, 2009.

[8] J. Li and N. Tian, "The M/M/1 queue with working vacations and vacation interruptions," Journal of Systems Science and Systems Engineering, vol. 16, no. 1, pp. 121-127, 2007.

[9] M. Zhang and Z. Hou, "Performance analysis of $M / G / 1$ queue with working vacations and vacation interruption," Journal of Computational and Applied Mathematics, vol. 234, no. 10, pp. 2977-2985, 2010.

[10] H. Zhang and D. Shi, "The $M / M / 1$ queue with Bernoullischedule-controlled vacation and vacation interruption," International Journal of Information and Management Sciences, vol. 20, no. 4, pp. 579-587, 2009.

[11] U. Yechiali, "Queues with system disasters and impatient customers when system is down," Queueing Systems: Theory and Applications, vol. 56, no. 3-4, pp. 195-202, 2007.

[12] E. Altman and U. Yechiali, "Analysis of customers' impatience in queues with server vacations," Queueing Systems: Theory and Applications, vol. 52, no. 4, pp. 261-279, 2006.

[13] N. Perel and U. Yechiali, "Queues with slow servers and impatient customers," European Journal of Operational Research, vol. 201, no. 1, pp. 247-258, 2010.

[14] S. Bocquet, "Queueing theory with reneging," Tech. Rep. DSTO-TR-1772, Defence Science and Technology Organisation, Defence Systems Analysis Division, Edinburgh, Australia, 2005. 
[15] P. V. Laxmi, V. Goswami, and K. Jyothsna, "Analysis of finite buffer Markovian queue with balking, reneging and working vacations," International Journal of Strategic Decision Sciences, vol. 4, no. 1, pp. 1-24, 2013.

[16] D. Yue, W. Yue, and G. Xu, "Analysis of customers' impatience in an $M / M / 1$ queue with working vacations," Journal of Industrial and Management Optimization, vol. 8, no. 4, pp. 895-908, 2012.

[17] N. Selvaraju and C. Goswami, "Impatient customers in an $M / M / 1$ queue with single and multiple working vacations," Computers \& Industrial Engineering, vol. 65, no. 2, pp. 207-215, 2013.

[18] J. Keilson and L. D. Servi, "A distributional form of Little's law," Operations Research Letters, vol. 7, no. 5, pp. 223-227, 1988. 


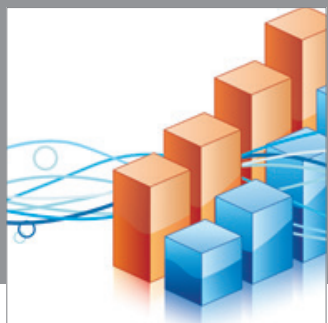

Advances in

Operations Research

mansans

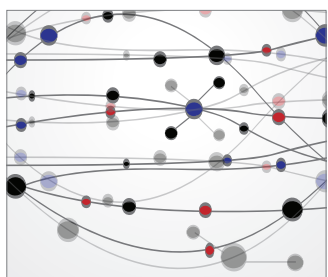

The Scientific World Journal
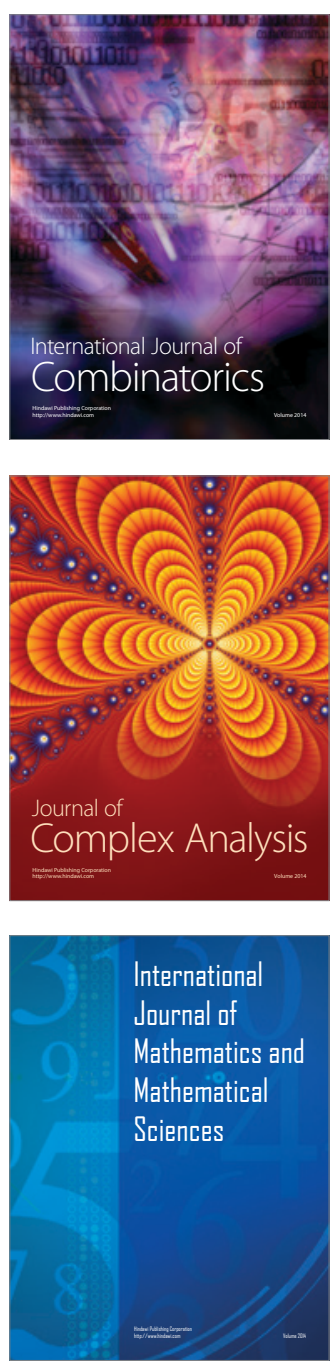
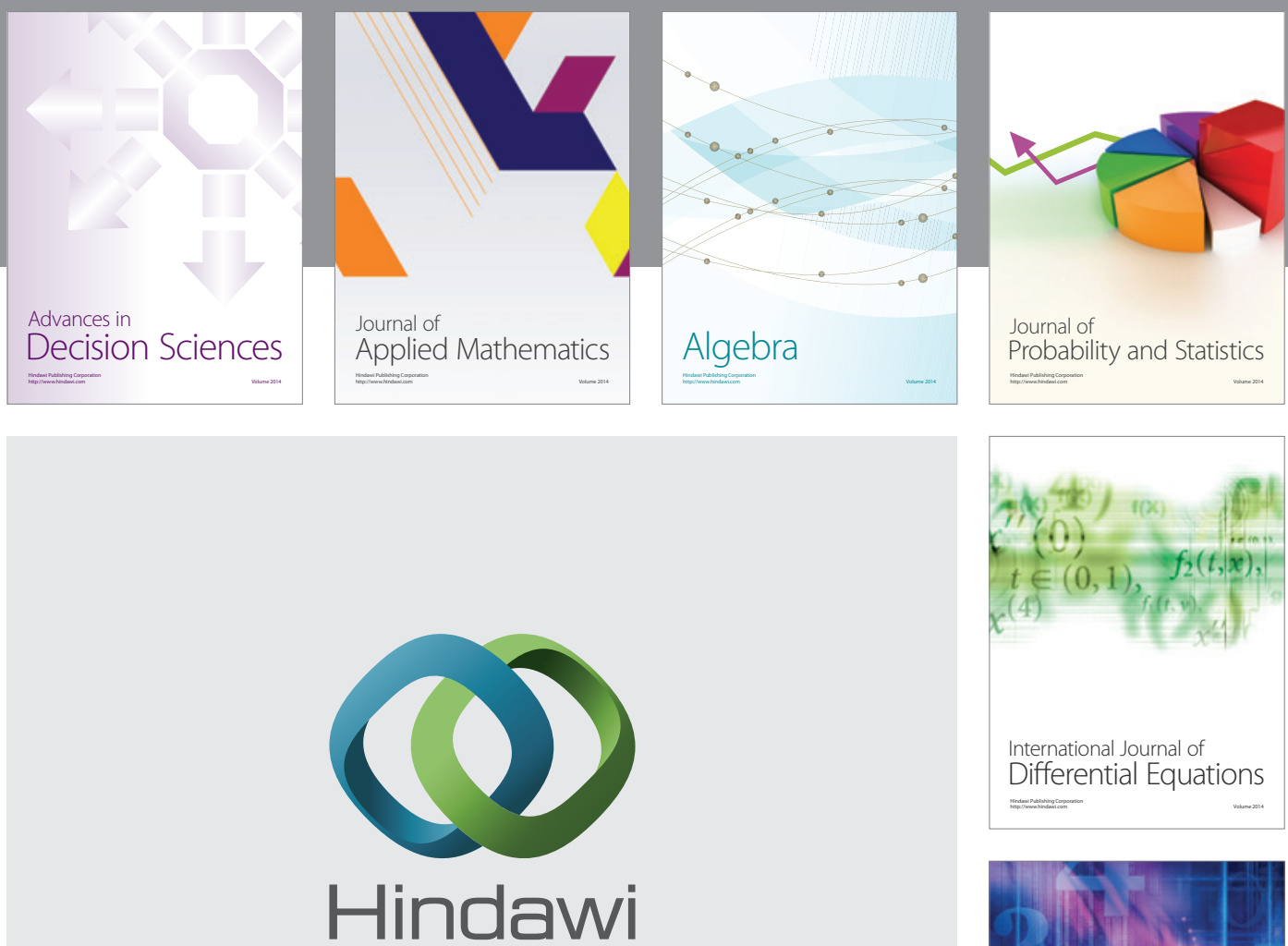

Submit your manuscripts at http://www.hindawi.com
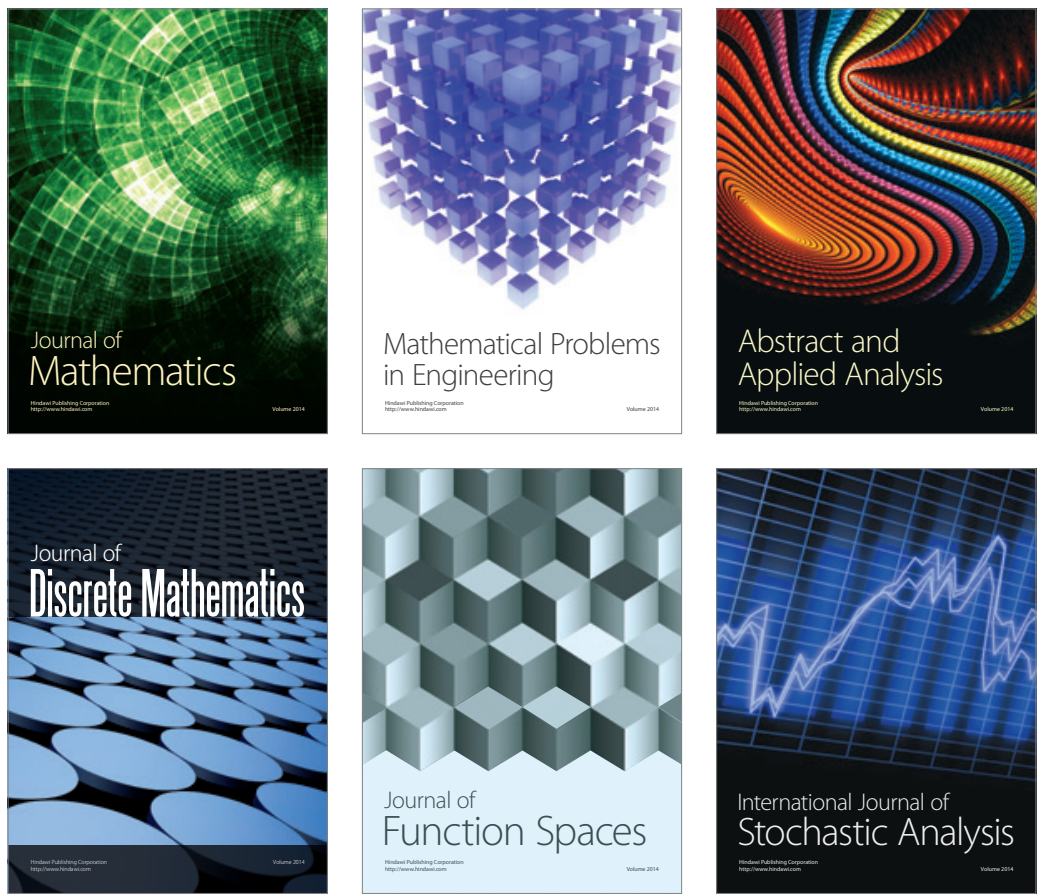

Journal of

Function Spaces

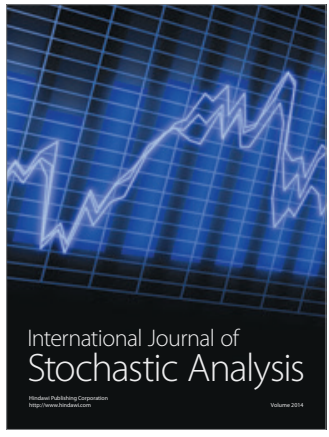

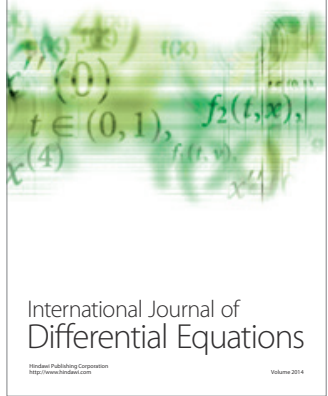
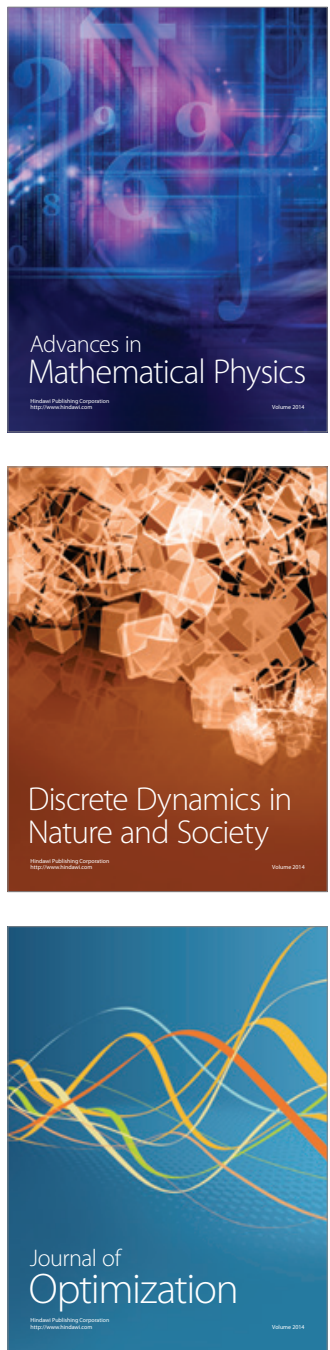